\title{
Preface: Special Issue-Frontiers in THz Technology II
}

\author{
Toshitaka Idehara $^{1} \cdot$ Norihisa Hiromoto $^{2}$. \\ Kiyomi Sakai $^{1,3} \cdot$ Masahiko Tani $^{1} \cdot$ Keisuke Tominaga $^{4}$
}

Received: 6 September 2016 / Accepted: 19 September 2016 /

Published online: 21 October 2016

(C) Springer Science+Business Media New York 2016

It has been almost two decades since the era of rapid progress on the terahertz $(\mathrm{THz})$ technology research in the latter half of the 1990s, and 3 years have passed since the First International Symposium on Frontiers in THz Technology (FTT 2012) was held in Nara. The research and development $(\mathrm{R} \& \mathrm{D})$ on the $\mathrm{THz}$ technology continues to make progress and to diversify from the basic research areas such as sources, detectors, components, materials, spectroscopy, and imaging, to the applied technologies in the areas of bio-medicine, industry, security, remote-sensing, communications, etc.

The Second International Symposium on Frontiers in THz Technology (FTT 2015) was organized in order to confirm the issues of the THz technology and to invent the applications that truly contribute to the human society. It is aimed assisting steady development of the research for the future by scoping the results and the products performed until now, searching for the important fields and the lodestar of the research and development, and by carefully watching the future trend including cutting-edge studies and new research seeds.

After closing the symposium successfully, we are organizing to publish the special issue: FTT 2015-Frontiers in THz Technology II with excellent papers in both THz Science and Technology areas. We hope our active contributions do bear fruit as excellent papers published in Journal of Infrared, Millimeter, and Terahertz Waves (JIMT).

Symposium chair: Norihisa Hiromoto

Guest editors: Toshitaka Idehara

Kiyomi Sakai

Masahiko Tani

Keisuke Tominaga

Toshitaka Idehara

idehara@ fir.u-fukui.ac.jp

1 FIR Center UF, University of Fukui, Fukui-shi, Japan

2 Shizuoka University, Hamamatsu-shi, Japan

3 NICT, Koganei-shi, Japan

4 Kobe University, Kobe-shi, Japan 